\title{
Four narratives about online international students: A critical literature review
}

In the current higher education context, where there is a growing economic imperative for universities to recruit more international students, offering online programmes is seen as an effective international recruitment strategy. However, supporting online international students studying at a distance is not a simple task for both universities and tutors. The problem mainly stems from a lack of theoretical understanding of the actual ways, in which online international students experience and engage with online learning. The present article, therefore, aims to address a gap in our current understanding of online international students, by systematically, yet critically reviewing relevant academic narratives about who online international students are. Our review reveals four types of narratives presented in the published academic literature, describing and discussing online international students in particular ways such as: i) unspecified others with a rapid increase in their numbers; ii) specific others with deficits; iii) specific others as pedagogical resources; and iv) active participants in international learning communities. We discuss both the merits and the drawbacks of each type of narratives for online educators seeking pedagogical suggestions about supporting online international students in their real-life teaching contexts.

Keywords: online international student; international education; international student; non-Western student; online higher education; critical literature review

\section{Introduction}

This article reports the results of a critical literature review of scholarly narratives about international students in online higher education (HE) settings. There has been a growing emphasis, in the increasingly competitive and market-driven HE context, on effective internationalisation strategies, which are seen as crucial for the success (or even survival) of HE institutions (Bourn, 2011; Warwick and Moogan, 2013). It is not a new phenomenon for universities to recruit students from overseas and bring them into their campuses. In recent times, however, it has become an economic imperative for $\mathrm{HE}$ institutions to recruit more international students; and, in turn, to accommodate their unique needs by providing more flexible ways of accessing university education when abroad (Monk, et al., 2015). Offering online programmes has been explored as an innovative mechanism posited to underpin effective internationalisation strategies - enabling international students to earn a foreign degree without leaving their home countries (HM Government, 2013; OLTF, 2011).

The nature of "learning at a distance" offers international students unique learning and living conditions, allowing their simultaneous presence in both overseas learning and home living spaces. That is, the "double internationality" of online international students, studying abroad but in their own country of residence, makes their learning experiences markedly different from those of campus-based international students-whose main challenges (e.g., cultural dislocation, social exclusion, and legal circumscription of political-economic status) normally arise from their physical re-location to an overseas campus. Yet, despite the proliferation of online programmes, and of international students being recruited to those programmes, little is known about the characteristics of those international students and their experiences of participating (Fenton-O'Creevy and van Mourik, 2016). Furthermore, the actual ways in which online international students' experiences are different from (or similar to) those of campus-based international students, and indeed those of other online students, seems to be unknown.

We suggest that the lack of such knowledge about online international students is, at least partially, due to a long-held assumption that embracing the open and inclusive nature of online communication will make $\mathrm{HE}$ provision more accessible. Such narratives tend to focus on the pedagogical possibilities of online technology rather than the distinctive needs of learners. From its birth, online HE has been assumed to provide equal opportunities, to various student groups, to participate in democratic group communication and collaborative learning activities (see Harasim, 2000). Yet, against the backdrop of the favourable climate for a rapid growth in online HE provisions and student numbers, the uniqueness of international students has been neglected or underrepresented in online HE literature. Studies concerning online students frequently conceptualise them as a 
homogenous group and offer general accounts of student experiences, which underpins a lack of appreciation of the diversity of online students in current HE contexts (Lee, 2017).

Research emerging in the context of language education has also stressed the great potential that online education has for non-native language speakers: for example, Freiermuth (2001) argues that both native and non-native speakers have more equal voices in online learning environments, while Kim (2011) suggests that non-native speakers in online contexts often engage in academic socialisation more actively than do their native peers. Despite the importance of those findings, arguments of that nature can serve to encourage a restricted focus on online international students' language competences or communicative behaviours. Similarly, in this context, online HE providers have failed to perceive online international students as more than simple recipients of the 'study abroad' opportunities that they offer: non-native speakers are positioned as depersonalised targets for recruitment into online programmes, and subsequently enumerated as indicators of successful outcomes for internationalisation strategies.

The starting point for our article is the opposite. We - as online tutors who have been interacting with a growing number of international students in our distance programme, as well as scholars in the field-perceive online international students as holistic human beings, coming to us with living and learning conditions both unique and complex. We also conceive the notion of internationalisation as an ongoing process, in line with the view of Warwick and Moogan (2013):

Internationalization extends beyond recruitment - it is an ongoing process, encompassing teaching and learning, research collaborations, curriculum development, the student experience, staff development, student support services and much more. (p. 118)

This extended view on internationalisation obligates us to recognise that there is a pedagogical imperative - distinct from the economic imperative, with its focus on attracting more international students - to better understand online international students and their learning needs and experiences. We aspire, consequently, to better serve those students: as educators rather than merely as recruiters.

The present article contributes towards developing a more holistic understanding of online international students. The underlying aim of our work was to collect the different academic narratives presented in currently published literature. This paper examines each of those narratives and poses a set of questions in relation to each: "what has been stated and what has not been stated about online international students in the selected literature?" and "to what extent are those statements effectively supported by evidence?"

Below, we categorise the narratives and provide a critical commentary on both the values and limitations of each, in the context of an urgent need to better understand online international students. That commentary will doubtless be perceived as subjective by comparison with prevalent business-oriented narratives about how to better recruit international students to universities. Nonetheless, we hope the paper will be of interest to other online HE providers and educators who share our frustration at the absence of meaningful education-focused conversations about how to better serve online international students.

\section{Methodology}

The review project followed a systematic scoping process when searching for literature and selecting articles for critical review (cf. Arksey \& O’Malley, 2005). The evidence base was collected by searching peer-reviewed journal articles and book chapters in Scopus, the largest abstract and citation database of peer-reviewed literature (www.scopus.com). The search was conducted based on the title, abstract, and keywords of papers, using the following compound search terms:

- “internationalisation" OR "globalisation" OR

- “international student/learner” OR "overseas student/learner” OR “Asian/African/American/Chinese/Japanese/Korean/Taiwanese... student/learner” AND

- "online/distance/virtual/open education/learning/course/program*/universit*” 
The above search, when conducted in January 2019, returned 418 items. Based on a discussion between the two authors, and with some input from a doctoral student whose thesis project is pertinent to the topic, inclusion/exclusion criteria were developed for a subsequent filtering process. That is, to be included in the review, we decided that the abstract of a paper must demonstrate: i) a focus on higher education; ii) a focus on pedagogical practice (rather than administrative matters, including recruitment); and iii) a focus on international students resident in a country different from the institution offering their online course.

To begin with, it should be noted that there has been relatively little research conducted on the topic of international students in online HE, especially by comparison with the many thousands of studies about online HE that do not categorise or label the students they study. In particular, when it comes to research outputs published through more reliable academic sources (in this case, our proxy measure was the peer-reviewed journals or books included in the Scopus database), the number of available texts was disappointingly small. After applying our filtering criteria to the initial 418 items, only 39 were selected for inclusion in the critical analysis.

We took a grounded theory approach (Charmaz, 2014) to analysing the selected papers, which were treated as an empirical data set for our project. The initial coding was done by the first author, following the guidelines suggested by Strauss and Corbin (2015), who propose three steps: open coding, axial coding, and selective coding. Firstly, each article was broken down into a series of meaning units of analysis (i.e., sentences or paragraphs defining and describing international students in online HE), with those units carefully coded. Our two dimensions for the initial coding schemes consisted of: claims (or statements) about international students in online HE, and the evidence presented to support the claims. Most articles presented multiple claims and the initial codes $(\mathrm{N}=61)$ were identified and named by highlighting meaningful phases on the printed articles, such as "internationalisation", "the silent Chinese", "English language support" and "group activities". The first author also made notes of potential categories emerging from the highlighted parts in the margins of the article.

The second round of reading (i.e., axial coding) was also undertaken by the first author, with the codes are more carefully examined and compared with/against each other at this stage. An attempt was made to identify and categorise claims and evidence that appeared more commonly, or which was ascribed more weight within the source material. As a result, the codes were logically organised and grouped into independent categories $(\mathrm{N}=9)$ : student numbers, educational necessities, skill deficits, learning difficulties, global connection, intercultural communication, programme limitations, practical suggestions, and pedagogical support. This coding exercise was documented within a spreadsheet and checked by the second author. Finally, both authors collaboratively conducted the process of selective coding and four - more substantial — themes were generated as a result. Those four themes (i.e., narratives about online international students) will be presented in the following section of this paper, with the exposition highlighting questions like "how are international students conceptualised by the theme?" and "how are characteristics of international students discussed by the theme?"

In view of the difficult nature of demonstrating the reliability and validity of qualitative research to others, the review study utilised the concept of "critical friends" as a mechanism for bolstering the "trustworthiness" of the research outcomes (cf. Lincoln \& Guba, 1985). The two authors initially analysed the texts, as described previously; and then engaged in conversations with two other colleagues (including the doctoral student mentioned above) to consider the themes to be pursued further and to construct the final narratives presented in this paper.

\section{Results}

In this section, we present the results of the analysis. We organise the exposition around four types of narratives: i) Unspecified others with a rapid increase in their numbers, ii) Specific others with deficits, iii) Specific others as pedagogical resources, and iv) Active participants in international learning communities. The aim is to unfold and present, in an organised and accessible manner, a range of common narratives about who online international students are and what experiences online HE provides to them. The numbers presented next to the title of each narrative theme indicate the total number of papers, out of the selected 39, in which the particular narratives appear in a strong form (most papers, as mentioned above, present more than one narrative). Below 
Lee, K., \& Bligh, B. (2017). Four narratives about online international students: A critical literature review. Distance Education. https://doi.org/ 10.1080/01587919.2019.1600363

the title for each theme we provide three examples of associated claims taken from the papers in our dataset, chosen to illustrate concretely how the narrative is constructed and presented in the academic literature.

\section{Unspecified others with a rapid increase in their numbers $(N=27)$}

Today in English-speaking countries, international students comprise substantial proportions of total enrollments [...] In 2011-2012, approximately $20.4 \%$ of the total number of international students registered with a higher education institution in the United Kingdom were studying through some form of flexible, distance, or open learning and were based overseas (UK council for International Student Affairs, 2013b). (Ramanau, 2016, p. 2)

The course was designed to attract both UK/UE and international students enabling them to remain in their own countries, while undertaking the programme. This paper is a case study and explores student experiences of learning alongside students from other countries among students registered on the course in academic year 2010-2011. (Gemmell, Harrison, Clegg, and Reed, 2015, p. 137)

The advancement of computer-mediated communication and Internet technology has shaped the landscape of higher education and allowed universities and educational institutes to expand their global outreach [...] Developing countries in Asia, including China and India, have been the most attractive destinations for offering online degrees because of their rapid rise in economic development and enormous demands for higher education access. (Liu, Liu, Lee, and Magjuka, 2010)

The first type of narrative about online international students is built around the affirmation of an increasing demand for HE access worldwide; the advancement of information and communication technology and increases in its educational use; and the economic and educational necessity of providing online HE to globally dispersed students. When expounding the narrative, most authors proceed by stating one or more of the following claims: i) that a growing need for HE access worldwide prompts a rising demand from international students, with online education an effective solution to meet it; ii) that there is a growing number of international students accessing HE institutions in Western (or English-speaking) countries via the means of online education; and iii) that internationalisation is fundamentally beneficial to both universities and students, including domestic students, living in a global society.

Making such claims does not usually involve providing specific information about who is being referred to as international students. Online international students are often portrayed as if an imaginary group existing somewhere far from the authors' (or their institutions') countries - in exotic lands given only generic descriptors: developing countries, non-Western countries, or Asian countries. One thing, however, is made clear about this unspecifiable mass - that they want to access HE provided by universities geographically located in developed countries or Western countries, to which they cannot physically attend. Those exotic others, therefore, have chosen to attend Western universities by registering for online programmes in great numbers, with even more of them expected to do so in the near future.

The immediate pedagogical implications set up by such assertions include the necessity of "satisfying" those unknown but significant others (presumably, to increase, or at least secure, their numbers) and the need to provide appropriate support for their distant populations. Furthermore, this type of narrative urges online tutors to design a more inclusive and diversified curriculum: one that is culturally relevant to today's multicultural student groups, including international students, and that is effective in facilitating interactions between international students and their domestic counterparts.

Although we consider that there is reasonable content within these assertions (especially the importance of bringing a 'global perspective' into online curricula), such narratives have stark limitations when considered from the perspective of online educators. The narrative carries a strong imperative flavour but is not, most of the time, based on considerations of actual students and specific pedagogical strategies. In essence, the narratives in 
this category, as found in the 25 papers, are largely rhetorical and frequently invoked within initial statementsused within the texts to emphasise the importance of the authors' articles and/or the initiatives presented in the papers. The assumptions in those narratives tend to be simply taken-for-granted by the authors, rather than being substantially discussed or articulated.

The evidence or data provided to support these narratives typically includes descriptive statistics, such as student numbers and the annual growth in those numbers - as the first example above shows (Ramanau, 2016). The second example - Gemmell et al. (2016) - also presents statistical data (e.g., the percentage of international students in UK postgraduate programmes) in other parts of the article. In addition, most articles providing such imperative narratives refer to particular national policies and related discussions, as the following excerpt from the second example above demonstrates:

Historically in the UK, internationalisation in higher education was seen in the context of international students who came to British universities to gain British education (Luxon \& Peelo, 2009). However, in the early 1980s government policy encouraged British universities to recruit more international students recognising that Britain lagged behind North American and Australian universities in this respect.

(Gemmell, et al., 2016, p. 138)

In some cases, these narratives are used as a "mantra", or a rehearsal of general claims about modern society in forms commonly used in business magazines or institutional strategic plans. For example, the third example above - that taken from Liu et al. (2010) — is presented as the very first paragraph of the paper without any supporting evidence and citations. The relation of these narratives to online HE is, perhaps, to exhort educators to accept the urgency of doing "something" better for those unspecified "others" - perhaps to exert pressure on individual educators to better cope with rapidly changing HE contexts. For that reason, these narratives tend to be provided as a frame for other types of narratives, rather than being core ideas of the papers.

As online educators, it is likely that such narratives might prompt questions, in turn, about who this unknown mass really are, how they are to be supported, how online tutors are to support their particular international students, and how those online tutors are supposed to know what has been effective or appropriate. The importance of "having" strategies is emphasised within this narrative, but the strategies themselves are not forthcoming from within it. In other words, this way of talking about online international students does not offer much in the way of meaningful pedagogical suggestions that might be recognised or enacted by those online educators seeking more effective ways to support their students.

\section{Specific others with deficits $(N=20)$}

International online students are expected to bring to the course an adequate knowledge of English. However even when a satisfactory [English] score is required to register for a course, many international students are unable to achieve parity in discussions and other written assignments with speakers whose first language is English. International students are also at risk of misinterpreting postings and assignments or of being misinterpreted by others thanks to misused vocabulary or grammar structures. (Sadykova and Dautermann, 2009, p. 98).

Findings indicate that previous education and especially language proficiency strongly impacted the learning of these students in this environment. Non-native English speakers required considerably more time to process readings and postings and to make postings themselves. Their lack of familiarity with the details of North American culture and colloquial language made it difficult to follow much of the course discussions. They also tended to avoid socializing in the course, which left them at the periphery of course activities (Zhang and Kenny, 2010, p. 17).

Much of this learning is implicit rather than explicit; acquired through example and feedback. Nonetheless, such genres and registers do not stand alone, but bear family resemblance to others rooted in the same traditions and social and cultural contexts [...] International students often share much less of the tacit knowledge required to engage successfully in the particular genres of 
academic writing required in UK higher education. In particular, as we discuss below, terms like 'critical' or 'reflective' are freighted with tacit meaning in certain academic genres and characterise practices which are signalled by specific choices of register in academic writing (Fenton-O'Creevy and van Mourik, 2016, p. 132).

The second type of narrative we identify shifts the focus to international students themselves - and immediately serves to highlight how international students may be different from their domestic counterparts. The narratives of this type, furthermore, conceptualise one or more deficits with which those international students are perceived to be associated, particularly those from non-Western countries. As the first example above — Sadykova and Dautermann (2009) — demonstrates, online international students are often stigmatised as "being at risk" of being unsuccessful or unsatisfactory in their learning. A deficit of language proficiency among online international students is the mostly frequently mentioned factor, with that deficiency recognised as likely to cause various challenges and difficulties throughout the learning process (see also Cong and Earl, 2011; Kwon et al., 2010).

It is also commonly suggested that online international students suffer from another deficiency: one related to the cultural understanding of (or familiarity with) what is expected in Western educational contexts. Specific groups of students are positioned as being passive, conformist, uncritical and silent (see also Liu, et al., 2010; Ramanau, 2016). It should be emphasised that not all of these narratives opt for the terminology of "deficiency". In other cases, an appeal to prior experiences or background is made, thereby suggesting a backdrop to the difficulties of international students' in making transitions to course practices and fully participating (see Liu and Magjuka, 2011).

Overall, from the vantage point of the present review, what is positive about these deficit narratives is that — by contrast to the imperative narratives discussed above - they highlight actual problems that have been experienced by particular student groups (e.g., Chinese, Russian, Korean, Asian, or African students) in particular online HE settings. Also, many of the relevant articles that do use these narratives as a basis for offering pedagogical solutions to identified problems: often, more or less specific strategies to support those international students with whom their narrative has been concerned. For example, Zhang and Kenny (2010) suggest three ways in which universities can improve the quality of international student learning experiences in their online programmes:

1) raise the English language proficiency requirement for graduate admissions into online programs because the text-based communication in a [computer-mediated communication] space requires interpreting messages without non-verbal cues; 2) ensure that online distance education course designers are aware of the needs and expectations of international students; and 3) combine the design principles from both traditional and constructivism theories. (p. 17)

Nevertheless, there are two interrelated assumptions that render these narratives problematic. Firstly, the formulation of deficits is commonly used to imply that there are definite divergences between domestic and international students in online HE; very often, that turns out to be a projected distinction between 'Western' and 'non-Western' students, a fact that is unproblematised by the authors and which is therefore, in our view, somewhat arbitrary. Secondly, homogenous assumptions about cultural and social background are frequently made on a national basis: it is assumed that there are distinctive characteristics shared among students from the same countries. For example, Zhang (2013) examines the influence of Confucian-heritage culture on Chinese learners' engagement in online discussion in U.S. HE. Based on observing the engagement patterns of 12 students from China, Taiwan, and Hong Kong, the author concludes that

As a result, when encountering difficulties in learning, the Chinese learners were intimidated to interact with their instructors. Instead, they tended to seek help from peers, particularly those who shared similar cultural and linguistic backgrounds. (p. 238).

Significant questions can be posed in relation to such statements, such as: how do researchers know and determine which students are from "Confucian traditions"; and can it be assumed that all students from China, 
Taiwan, and Hong Kong (each with distinctive historical and societal characteristics, and the former a very large-scale geographical construct) are from the same cultural background? To address the same issue from a different angle, we might consider whether we would think it acceptable to assume that all Western students had shared attitudes towards their instructors and peers, ones fundamentally different from Chinese students. This issue seems increasingly urgent given the growing recognition of diversity and multiculturalism within societies across much of the globe.

Of course, we should emphasise that appreciating the potential distinctions between international and non-international students is not an issue in itself. As the examples above suggest, these narratives are often based on empirical data, collected from actual international students. However, it is a real issue if an imprecise conceptualisation of distinctions between students serves to overgeneralise narratives about who particular groups of international students are and what their learning experiences look like - typically in negative ways. Some studies projecting this type of narrative are in danger of disregarding that "learning at a distance" can, by itself, be a challenging task for all—even for domestic students (see Lee, 2017). Thus, the validity of the empirical warrant for these deficit narratives can be critiqued methodologically, on the basis that the difficulties of non-international students in the same settings have typically been obscured.

It is noteworthy that all 20 papers that project these narratives share the same rhetorical structure: firstly, pre-determined sets of difficulties that international students may face in their online learning process are established; secondly, a consequential need for extra support is validated. The extra support being suggested takes various forms, but generally tends to have a "remedial" quality, directed at the particular shortcomings earlier emphasised by the authors. One outcome of such a logic is that the sense of international students having a degree of agency to pursue their own development is underplayed. Furthermore, this type of narrative fails to recognise some of the useful affordances that online HE may provide to international students — with honourable exceptions, such as the one below:

Online environment may provide Chinese learners, particularly those whose first language was not English, with more privacy and additional time to prepare for class discussion by removing barriers of being embarrassed or being shy. (Zhang, 2013, p. 250)

Thus, despite their positive aspects, these deficit narratives fail to provide online educators with balanced and sophisticated views about what it means to be an online international student, or how tutors might support such students in their courses. At their worst, narratives of this type can serve to reinforce those preconceptions and biases that online tutors might already have regarding different ethnic groups and/or nationalities.

\section{Specific others as pedagogical resources $(N=8)$}

The internationalization and globalization of education have been brought up through the increasing demand for appropriate education to achieve better understanding, analyzing and responding to the unpredictable and changing situations and crossing the geographical borders among the nations to establish an international community for better social, occupational and emotional life; therefore, higher education institutions need a different approach to respond to these educational demands qualitatively and quantitatively; they value the participation of international students (Vajargah \& Khoshnoodifar, 2013, p. 147)

One of the key drivers for the development of this module was to provide access to an authentic international learning experience for students who did not have the opportunity to experience overseas clinical placements due to the many social and financial pressures they face [...] The majority of students appreciated the opportunity to engage directly with international peers without having to travel as a distinct advantage of this module (Strickland, Adamson, McInally, Tiittanen, and Metcalfe, 2013, p. 1163)

Students on an online distance learning programme benefit from learning alongside international students through learning about the experiences of health professionals and health 
systems in other countries and though appreciation of other perspectives and the importance of the context of public health issues. Strategic use of a wide range of learning tools and technologies that increase collaborative working such as wiki's, blogs and discussion boards enhances the benefits of internationalisation (Gemmell, Harrison, Clegg, \& Reed, 2015, p. 145)

The third type of narrative highlights the pedagogical value of intercultural exchanges, and of learning about other "cultures" through interacting with international students. Texts incorporating this type of narrative often proceed from favourable notions of international education and internationalised curricula, positioning them as an essential component of contemporary HE provision. Those notions-already nascent in the preceding, numbers- and deficit-oriented, narratives - are here strongly emphasised, set up as both intrinsically positive and important for the future of students and the HE sector alike. The first example above - taken from Vajargah \& Khoshnoodifar (2013) —illustrates this tendency. Narratives of this type share a sense of urgency about preparing students for participating in a global economy as competent workers; doing so will be achieved, it is suggested, by providing them with a range of educational opportunities to develop global perspectives and knowledge, as well as intercultural communication skills.

One striking finding from our analysis is that the main focus of these narratives is often not on international students but, rather, on their domestic counterparts. Such counterparts often have an identified need to access international learning opportunities without leaving their own institutional context - that is, without directly immersing themselves in international situations. This type of narrative, therefore, positions international students as pedagogical resources whose presence in online HE serves to make learning environments and curricula 'internationalised'. The following student interview, excerpted from Strickland et al. (2013), sets up such a vantage point, whereby international students are seen as having instrumental or economic value for providing internationalised learning:

Traditionally, international opportunities are undertaken as expensive elective placements, out of the reach of many students. By undertaking this online module, I have been able to develop a strong understanding of the issues and challenges faced by US nurses, an opportunity that would not have otherwise have been open to me. Wider access to international opportunities is important. (p. 1164)

In these narratives, therefore, international students are specific others becoming pedagogical resources within online HE. Another striking finding, related to the preceding point, is that these value-oriented narratives most commonly arise in papers describing or evaluating specific educational initiatives: ones that create international connections between two more courses or universities across different countries. One such initiative is described in the following way:

International partnerships for this module were developed from existing networks. Partners from Western Carolina University (USA) and Lahti University of Applied Sciences (Finland) were involved in negotiating the learning activities to ensure a comparable learning experience for all students [...] Students collaborated through the wiki for an 8 week period during this trimester with a total of 22 students from the three participating institutions: 8 from USA, 7 from UK and 7 from Finland (Strickland, et al., 2013, p. 1161)

In some respects, such value-laden narratives resemble the imperative narratives of our first theme in that they establish a sense of urgency in the internationalisation of online teaching practices. However, unlike those imperative narratives, which fail to specify international students beyond simple numbers, narratives of the present type are constructed out of empirical studies involving specifiable international students (the " 8 from USA, 7 from UK and 7 from Finland" in the above example). As the examples at the beginning of the section illustrate, the narratives go on both to celebrate the success of the initiatives they evaluate, and to provide illustrative data indicating positive results about learning satisfaction, behaviours, and outcomes - tellingly, often taken from the perspective of domestic students. 
In comparison with the first theme, then, implications for educational practice can often be inferred more directly from narratives of this type. However, from an educator vantage point, there are at least two significant shortcomings.

The first of those shortcomings is that these narratives are often not focussed on the complexity (or the multi-voiced nature) of the educational phenomena they highlight. Intercultural communication-and specifically online intercultural communication - is a challenging and disruptive situation even for experienced researchers with willing international collaborative partners. As one of the present authors has noted elsewhere, providing students with international learning experiences is a challenging task for individual tutors where they cannot leverage considerable social and linguistic capital (Lee, 2018). It is worth emphasising that in the above example, Strickland, et al. (2013) utilised existing international networks to develop their online module. Not all online tutors, of course, would have those "existing" networks.

If the flagship programmes being evaluated by those papers projecting these narratives are reliant on support infrastructure to which most, ordinary, online educators might not have access, then that might obviously influence how we interpret the narratives themselves. In addition, by narrowly focusing upon and promoting positive outcomes, these resource-oriented narratives serve to downplay more critical thoughts about the challenging nature of the learning and teaching activities being suggested, particular for international students. It is certainly difficult for a readership of online educators in general settings to connect to their own local practices the noble findings and subsequent recommendations that are presented.

The second shortcoming we perceive, a concern about the stratification among different groups of international students, builds upon the first. By comparison with the second theme (Specific others with deficits), these resource-oriented narratives do laudably position international students as the equal counterparts of domestic students. Indeed, in some cases, narratives of this type further blur the distinction between "international" and "domestic" students — on the grounds that both groups need to acquire intercultural communication skills, and that both groups are "needed" to create meaningful intercultural communication opportunities.

It is worth noting an assumption underpinning this comparatively radical elevation of the status of international students: it is possible because participating international students are fully capable of engaging in the communicative activities. That is a starkly different assumption from those narratives in the preceding theme. What, then is driving that difference? The answer can be found by recalling that international students in the deficit narratives were largely specified as non-Western and non-native speakers. The papers exhibiting the current narrative types, by contrast, make no such specification, and often explicitly describe situations that vary from that template - as Strickland, et al.'s (2013) description of their participants as being "8 students from USA, 7 from UK and 7 from Finland" makes perfectly clear.

To be clear, we perceive nothing wrong with practitioners being positive and excited about the potential benefits to students of having access to international learning opportunities; or about the fact that emerging telecommunication tools can create those opportunities at relatively low cost. The opportunities themselves are very real. However, our analysis does highlight a need to be more cautious about constructing deficit and value narratives. By placing those two narratives in parallel and highlighting how they each position particular groups of international students, we hope to illustrate that a double standard is being constructed. This double standard may create or reinforce destructive, rather than supportive, attitudes towards international students among online educators and students: attitudes whereby distinct groups of international students are treated differently, according to the instrumental value they bring into online learning settings.

\section{Active participants in international learning communities $(N=6)$}

International students taking an online course will encounter many challenges, but there are steps that can be taken to help make their learning experience more successful [...] Many international students may find it demanding and challenging at first, but participating will help build their confidence and assist in the creation of relationships. It makes a big difference for the 
international student when he or she realizes that others have going through the same processes as them and have the same questions and anxieties. (Chen, Bennett, and Maton, 2008, p. 983)

Peers become invaluable mediators of knowledge for international students who seek peer assistance to compensate for the lack of culture-specific knowledge and skills and to satisfy their interest in the host culture. The study suggests that course developers and facilitators should be proactive when assigning group projects and activities so as to enable close peer-to-peer interaction and opportunities for building personal relationships with other class members. (Sadykova, 2014, p. 24)

The aim of the study was to explore if the EdD international students' interactions and collaboration within the learning environment resembled an authentic online learning community of inquiry. In addition, we were interested in exploring whether the design of the program supported the evolution and elevation of EdD cohorts to online learning communities. (Crosta, Manokore, Gray, 2016, p. 47)

The fourth, final type of narrative suggests more constructive understandings of international students and their experiences: perceiving them as active participants within international learning communities, in which every single participant is unique regardless of their origins or locations. This comprehensive, more inclusive view of international students often proceeds from authors' critical reflection on their own interactions with those students. From the vantage point of online educators, we find these emancipatory narratives more helpful and insightful for two key reasons.

The first reason is that the narratives describe international students' online learning experiences, without unnecessarily generalising them (as having fixed characteristics) or being judgemental about what is observed and described. These accounts, thereby, allow for a greater possibility of noticing development or change-in the texts we found, that development most typically relates to students' gradual accommodation to online learning environments or to intercultural communication practices.

For example, the first illustrative excerpt above - that taken from Chen, Bennett, and Maton (2008) illustrates one moment within an in-depth description wherein two particular Chinese students themselves proactively addressed some of the challenges they faced when participating in an online course offered by a university in Australia. The authors subsequently expand that focus, to conceptualise the adaptation processes of international students as they encounter a variety of challenges within an online learning environment. In Chen, Bennett, and Maton's study, international students are not simply disadvantaged, needing support from other parties (such as their domestic peers, tutors, and universities); instead, they are active agents in their own learning processes who attempt to develop their own coping strategies and skills.

Secondly, the texts maintain a relatively practical essence to their narratives; typically doing so by emphasising what online educators actually did (rather than merely 'should do') when designing an individual module or working to unfold a set of teaching-learning interactions. Furthermore, the actions described often highlight the integration of pedagogical practices and strategies across courses and students - both international students and their domestic counterparts - rather than focussing on providing remedial support for targeted individuals or groups. The essential difference between the resource-oriented narratives above-in which international students are a means to achieve the specific goal of international education - and these more emancipatory narratives is this: here, both international education and students are each perceived as the natural educational background and conditions for online higher education.

For narratives built on such assumptions, there seems no particular need to problematise international students (or, indeed, to celebrate their presence). The narratives in the 6 papers we highlight under this theme are more inclined, instead, towards developing a comprehensive understanding of what is going on in particular modules experiencing growing diversity in their participants. Sometimes that narrative is expressed more specifically, as being about and how to make the international learning community stronger, as Sadykova (2014) argues in our second illustrative example. Thus, this type of narrative is emancipatory in its nature but not only 
with regard to international students. In this type of narrative, both tutors and students (including international students themselves) are responsible for supporting international students' experiences and developing supportive learning communities.

Through the process of undertaking this analysis, it has become clearer, at least to us, that our ultimate focus should be on developing a stronger sense of community among our learners: perhaps by employing diverse pedagogical strategies to promote particular forms of peer-to-peer interaction in our module. That, to say the least, should not be a new idea to many online tutors. Yet it is also not an additional burden suddenly created by international students entering some given module. From this starting point, online educators can strive to develop a collective mind-set whereby students - both international and domestic ones - rely on each other to create an active learning community among themselves, where a key objective for student participation is to support others' successful learning experiences.

It is noteworthy that all texts featuring this type of emancipatory narrative tend to be very cautious about making definite, universal claims about international students. Furthermore, the texts' authors each strive to encompass vantage points from both domestic and international (or 'Western' and 'non-Western') students within single narratives, rather than viewing either set of students as a negation of the other. As Crosta, Manokore and Gray (2016) — in the third example, above — rightly point out, in an international cohort, all students are international to each other. By utilising such a perspective, narratives of this type are able to avoid universalising the deficits or instrumental values they attach to international students.

\section{Conclusion}

Parent, bilingual, veteran, part-time student, online student, scholarship awardee, international student, dual citizen, traveling professional: how frequently do you come across a student who is only one of these things? Fewer and fewer students are members of only one single category. Instead, students are living their lives at the juncture of identities, often creating their own salient "selves" that better represent the intersectionality of their lives.

(Gargano and Throop, 2017, p. 919, emphasis added)

The present article aims to address a gap in our current understanding of online international studentsby systematically yet critically reviewing relevant academic narratives about who online international students are. Our review has revealed four types of narratives present in the published academic literature; each has a different starting point, and unfolds to describe and discuss online international students in different ways. Online international students are identified, in turn, as: i) Unspecified others with a rapid increase in their numbers; ii) Specific others with deficits; iii) Specific others as pedagogical resources; and iv) Active participants in international learning communities. Our analysis has considered each type of narrative, in both its merits and drawbacks, from the vantage point of online educators (perhaps seeking pedagogical suggestions about supporting online international students in real-life teaching contexts) rather than that of business and recruitment.

Overall, our review highlights that the fourth type of narrative is of particular merit. That narrative proceeds by perceiving international students as a natural part of online teaching practice - as authentic beings with their own unique strengths and weaknesses, just like any other students in an online course. That supports a holistic view: one that focuses on developing a supportive international learning community in collaboration with our students. In turn, from such a view tends to emerge pedagogical ideas that are both more practical and more valid due to the contextualisation of their account. In addition, our review has highlighted an urgent requirement to hear more of the authentic voices of international students in their full particularity - doing so without pre-conceiving what they might want to say about themselves and their learning experiences. Understanding and responding to the particular challenges they face, and valorising the specific contributions they make to the learning community, can be intertwined and collaborative processes.

Through focusing on our daily interactions with all of our online students, rather than isolating the international, we may be able to escape from a sense of superiority - as Western educators solving problems for others with deficits. We might partially ameliorate an enormous sense of burden that arises when online 
Lee, K., \& Bligh, B. (2017). Four narratives about online international students: A critical literature review. Distance Education. https://doi.org/ 10.1080/01587919.2019.1600363

internationalised education is confronted as a solo performer, responsible for coming up with grand plans for global links that might make our teaching more international. In addition, we might be able to notice possibilities for taking small yet practical steps towards more inclusive online learning — in ways that take advantage of both contingent possibilities and wider pedagogical strategising. That sensitivity, in turn, can make actual changes in our teaching more likely - thus having a cumulative effect bigger than those of blunter, imperative narratives.

\section{There are no conflicts of interest.}

References

Arksey, H., \& O'Malley, L. (2005). Scoping studies: towards a methodological framework. International Journal of Social Research Methodology, 8(1), 19-32.

Bourn, D. (2011). From internationalisation to global perspectives. Higher Education Research \& Development, $30(5), 559-571$.

Charmaz, K. (2014). Constructing grounded theory. Sage.

Chen, R. T. H., Bennett, S., \& Maton, K. (2008). The adaptation of Chinese international students to online flexible learning: Two case studies. Distance Education, 29(3), 307-323.

Cong, Y., \& Earl, K. (2011). Chinese postgraduate students learning online in New Zealand: Perceptions of cultural impact. In Cases on Globalized and Culturally Appropriate E-Learning: Challenges and Solutions (pp. 73-93). IGI Global.

Crosta, L., Manokore, V., \& Gray, M. (2016). From an online cohort towards a community of inquiry: International students' interaction patterns in an online doctorate program. Journal of Interactive Online Learning, 14(2).

Fenton-O'Creevy, M., \& van Mourik, C. (2016). 'I understood the words but I didn't know what they meant': Japanese online MBA students' experiences of British assessment practices. Open Learning: The Journal of Open, Distance and e-Learning, 31(2), 130-140.

Freiermuth, M. R. (2001). Native speakers or non-native speakers: Who has the floor? Online and face-to-face interaction in culturally mixed small groups. Computer assisted language learning, 14(2), 169-199.

Gargano, T., \& Throop, J. (2017). Logging on: Using online learning to support the academic nomad. Journal of International Students, 7(3), 918-924.

Gemmell, I., Harrison, R., Clegg, J., \& Reed, K. (2015). Internationalisation in online distance learning postgraduate education: a case study on student views on learning alongside students from other countries. Innovations in Education and Teaching International, 52(2), 137-147.

Harasim, L. (2000). Shift happens: Online education as a new paradigm in learning. The Internet and higher education, 3(1-2), 41-61.

HM Government (2013). International education strategy: Global growth and prosperity. Department for Business, Innovation and Skills, London. Retrieved from https://www.gov.uk/government/publications/international-education-strategy-global-growth-andprosperity

Kim, H. K. (2011). Promoting communities of practice among non-native speakers of English in online discussions. Computer Assisted Language Learning, 24(4), 353-370. 
Lee, K., \& Bligh, B. (2017). Four narratives about online international students: A critical literature review. Distance Education. https://doi.org/ 10.1080/01587919.2019.1600363

Kwon, K., Han, D., Bang, E. J., \& Armstrong, S. (2010). Feelings of isolation and coping mechanism in online learning environments: A case study of Asian international students. International Journal of Learning, 17(2). 343-356.

Lee, K. (2017). Rethinking the accessibility of online higher education: A historical review. The Internet and Higher Education, 33. 15-23.

Lee, K. (2018). Implementing computer-mediated intercultural communication in English education: A critical reflection on its pedagogical challenges. Journal of Computer Assisted Learning, 2018, 1-15.

Lincoln, Y. S., \& Guba, E. G. (1985). Naturalistic inquiry. Beverly Hills, CA: Sage.

Liu, X., Liu, S., Lee, S., \& Magjuka, R. J. (2010). Cultural differences in online learning: International student perceptions. Educational Technology \& Society, 13(3), 177-188.

Liu, X., \& Magjuka, R. J. (2011). Learning in cross-cultural online MBA courses: Perceptions of Chinese students. In Cases on globalized and culturally appropriate e-learning: Challenges and solutions (pp. 168-186). IGI Global.

Monk, N., McDonald, S., Pasfield-Neofitou, S., \& Lindgren, M. (2015). Portal Pedagogy: From interdisciplinarity and internationalization to transdisciplinarity and transnationalization. London Review of Education, 13(3), 62-78.

OLTF (2011). Collaborate to compete: Seizing the opportunity of online learning for UK higher education: Report to HEFCE by Online Learning Task Force. Retrieved from http://www.hefce.ac.uk/media/hefce1/pubs/hefce/2011/1101/11_01.pdf

Sadykova, G. (2014). Mediating knowledge through peer-to-peer interaction in a multicultural online learning environment: A case of international students in the US. The International Review of Research in Open and Distributed Learning, 15(3).

Sadykova, G., \& Dautermann, J. (2009). Crossing cultures and borders in international online distance higher education. Journal of Asynchronous Learning Networks, 13(2), 89-114.

Ramanau, R. (2016). Internationalization at a distance: A study of the online management curriculum. Journal of Management Education, 40(5), 545-575.

Strickland, K., Adamson, E., McInally, W., Tiittanen, H., \& Metcalfe, S. (2013). Developing global citizenship online: An authentic alternative to overseas clinical placement. Nurse education today, 33(10), 11601165 .

Strauss, A. L., \& Corbin, J. (2015). Basics of qualitative research: Grounded theory procedures and techniques. London, UK: Sage.

Vajargah, K. F., \& Khoshnoodifar, M. (2013). Toward a distance education based strategy for Internationalization of the curriculum in higher education of Iran. Turkish Online Journal of Educational Technology-TOJET, 12(1), 346-359.

Warwick, P., \& Moogan, Y. J. (2013). A comparative study of perceptions of internationalisation strategies in UK universities. Compare: A Journal of Comparative and International Education, 43(1), 102-123.

Zhang, Y. (2013). Power distance in online learning: Experience of Chinese learners in US higher education. The International Review of Research in Open and Distributed Learning, 14(4). 
Lee, K., \& Bligh, B. (2017). Four narratives about online international students: A critical literature review. Distance Education. https://doi.org/ 10.1080/01587919.2019.1600363

Zhang, Z., \& Kenny, R. (2010). Learning in an online distance education course: Experiences of three international students. The International Review of Research in Open and Distributed Learning, 11(1), 17-36. 